\title{
Analysis of the One-dimensional Euler-Lagrange equation of continuum mechanics with a Lagrangian of a special form
}

\author{
E.I. Kaptsov ${ }^{\mathrm{a}, \mathrm{b}}$, S.V. Meleshko ${ }^{\mathrm{b}, *}$ \\ ${ }^{a}$ Keldysh Institute of Applied Mathematics, \\ Russian Academy of Science, Miusskaya Pl. 4, Moscow, 125047, Russia \\ ${ }^{b}$ School of Mathematics, Institute of Science, \\ Suranaree University of Technology, 30000, Thailand
}

\begin{abstract}
Flows of one-dimensional continuum in Lagrangian coordinates are studied in the paper. Equations describing these flows are reduced to a single Euler-Lagrange equation which contains two undefined functions. Particular choices of the undefined functions correspond to isentropic flows of an ideal gas, different forms of the hyperbolic shallow water equations. Complete group classification of the equation with respect to these functions is performed.
\end{abstract}

Using Noether's theorem, all conservation laws are obtained. Their analogs in Eulerian coordinates are given.

Keywords: Gas dynamics equations, shallow water equations, Lie group, group classification, conservation law, Noether's theorem

\section{Introduction}

Modelling physical phenomena in continuum mechanics is considered in two distinct ways. The typical approach uses Eulerian coordinates, where flow quantities at each instant of time during motion are described at fixed points. Alternatively, the Lagrangian description is used, where the particles are identified by the positions which they occupy at some initial time. Typically, Lagrangian coordinates are not applied in the description of fluid motion. In practice such description is often too detailed and complicated, but it is always implied in formulating physical laws [1]. However, in some special contexts the Lagrangian description is indeed useful in solving certain problems.

Many models in fluid mechanics presented in Eulerian coordinates can be considered as a particular class of the model described by the equations [2, 3]

$$
\begin{gathered}
\dot{\rho}+\rho \operatorname{div}(u)=0, \quad \rho \dot{u}+\nabla \partial=0, \\
\partial=\rho \frac{\delta W}{\delta \rho}-W=\rho\left(\frac{\partial W}{\partial \rho}-\frac{\partial}{\partial t}\left(\frac{\partial W}{\partial \dot{\rho}}\right)-\operatorname{div}\left(\frac{\partial W}{\partial \dot{\rho}} u\right)\right)-W,
\end{gathered}
$$

where $W(\rho, \dot{\rho})$ is a given potential, 'dot' denotes the material time derivative: $\dot{f}=\frac{d f}{d t}=f_{t}+u \nabla f$ and $\frac{\delta W}{\delta \rho}$ denotes the variational derivative of $W$ with respect to $\rho$ at a fixed value of $u$. In particular, the gas dynamics

\footnotetext{
${ }^{*}$ Corresponding author

Email addresses: evgkaptsov@gmail.com (E.I. Kaptsov), sergey@math.sut.ac.th (S.V. Meleshko)
} 
equations and the shallow water equations have the form (10). Equations (11) can be derived as Euler-Lagrange equations in Lagrangian coordinates [2, 3]. The representation of equations (10) in the form of the Euler-Lagrange equation allows one to use Noether's theorem for constructing conservation laws [4].

In one-dimensional case Eulerian coordinates $(t, x)$ and mass Lagrangian coordinates $(t, s)$ are related by the condition [1]

$$
x=\varphi(t, s)
$$

where the function $\varphi(t, s)$ is a solution of the equation

$$
\varphi_{t}(t, s)=u(t, \varphi(t, s))
$$

Here $u(t, x)$ is the velocity in Eulerian coordinates which is related with the velocity $\tilde{u}(t, s)$ in the mass Lagrange coordinates by the relation $[5]$

$$
\tilde{u}(t, s)=u(t, \varphi(t, s))
$$

In 6] it was shown that one-dimensional equations (1) are equivalent to an Euler-Lagrange equation with the Lagrangian of the form

$$
\mathcal{L}=\frac{\varphi_{t}^{2}}{2}-\rho^{-1} W(\rho, \dot{\rho})
$$

where $\rho=\varphi_{s}^{-1}$ and $\dot{\rho}=-\varphi_{s}^{-2} \varphi_{t s}$.

The one-dimensional hyperbolic shallow water equations with linear bottom are derived from a particular case, where $W=W(\rho)$. For a nonlinear bottom one has to assume that the function $W$ also depends on $x$.

The present paper is focused on Euler-Lagrange equations in Lagrangian coordinates, where

$$
W=\tilde{g}(\rho)+\rho^{-1} h(x)
$$

The functions $\tilde{g}$ and $h$ are arbitrary functions of their arguments. This case includes the hyperbolic shallow water equations in different forms, and the isentropic gas dynamics equations. Group properties of the latter equations in Eulerian coordinates were studied in [7, 8, 9, 10]. In [11, 12, 13, 14] group analysis was applied to the gas dynamics equations of a polytropic gas in mass Lagrangian coordinates. Using Lagrangian and Noether's theorem, authors of [12] constructed conservation laws for letter mentioned equations in isotropic case. For nonlinear case the same approach was used in [15]. Here we also should mention results of [16], where nonlocal conservation laws were obtained.

The objective of the present paper is to perform group classification of the Euler-Lagrange equation with respect to the functions $g, h$, where the Lagrangian has the form

$$
L=\frac{\varphi_{t}^{2}}{2}+g\left(\varphi_{s}\right)+h(\varphi) .
$$

Applying Noether's theorem, conservation laws are derived.

\footnotetext{
${ }^{1}$ Further we omit the sign ${ }^{\sim}$ as it is not ambiguous.
} 


\section{The studied equation}

Considering the Lagrangian (2), one derives the Euler-Lagrange equation $\frac{\delta \mathcal{L}}{\delta \varphi}=0$ :

$$
\varphi_{t t}+G \varphi_{s s}-H=0
$$

where $\frac{\delta}{\delta \varphi}$ is the variational derivative, $G \equiv g^{\prime \prime}$, and $H \equiv h^{\prime}$. In order to preserve hyperbolicity of equation (3) it is assumed that $G<0$.

In Eulerian coordinates, equation (3) reduces to the system of equations

$$
\begin{gathered}
\rho_{t}+u \rho_{x}+\rho u_{x}=0, \\
u_{t}+u u_{x}-G\left(\frac{1}{\rho}\right) \rho^{-3} \rho_{x}-H(x)=0 .
\end{gathered}
$$

In particular, for equations studied in [9]

$$
G\left(\frac{1}{\rho}\right)=-\gamma_{1} \rho^{2}\left(\rho+\gamma_{2}\right), \quad H(x)=0,
$$

and the second equation of (41) becomes

$$
u_{t}+u u_{x}+\gamma_{1}\left(1+\frac{\gamma_{2}}{\rho}\right) \rho_{x}=0 .
$$

Another particular case of equations (4) was analyzed in [10], where equations with a variable bottom $\chi(x)$ were considered. For this case

$$
G\left(\frac{1}{\rho}\right)=-\rho^{3}, \quad H(x)=\chi^{\prime}(x),
$$

and the second equation of (4) becomes

$$
u_{t}+u u_{x}+\rho_{x}-\chi^{\prime}=0
$$

\section{Group classification of equation (3)}

The first step in group classification consists of finding equivalence Lie group, which can be used for simplifications of group classification.

\subsection{Equivalence Lie group}

Equation (3) contains the arbitrary functions $G$ and $H$. Equivalence transformations saving a structure of equations are able to change the arbitrary elements.

A generator of an equivalence Lie group [7] is assumed to be in the form

$$
X^{e}=\xi^{t} \partial_{t}+\xi^{s} \partial_{s}+\eta \partial_{\varphi}+\zeta^{G} \partial_{G}+\zeta^{H} \partial_{H}
$$

where $\xi^{t}, \xi^{s}, \eta$ depend on $(t, s, \varphi)$, and $\zeta^{G}, \zeta^{H}$ depend on $(t, s, \varphi, G, H)$.

Applying the prolonged generator to the system consisting of equation (3) and the equations

$$
\begin{gathered}
G_{t}=0, \quad G_{s}=0, \quad G_{\varphi}=0, \quad G_{\varphi_{t}}=0, \\
H_{t}=0, \quad H_{s}=0, \quad H_{\varphi_{s}}=0, \quad H_{\varphi_{t}}=0,
\end{gathered}
$$


one obtains the following generators:

$$
\begin{aligned}
& X_{1}^{e}=\partial_{t}, \quad X_{2}^{e}=\partial_{s}, \quad X_{3}^{e}=\partial_{\varphi}, \\
& X_{4}^{e}=t \partial_{t}+s \partial_{s}-2 H \partial_{H}-\varphi_{s} \partial_{\varphi_{s}}, \\
& X_{5}^{e}=s \partial_{s}+2 G \partial_{G}-\varphi_{s} \partial_{\varphi_{s}}, \\
& X_{6}^{e}=\varphi \partial_{\varphi}+H \partial_{H}+\varphi_{s} \partial_{\varphi_{s}} .
\end{aligned}
$$

These generators define the equivalence Lie group of equation (3).

Notice that also there is the involution

$$
\varphi \mapsto-\varphi, \quad H \mapsto-H,
$$

which is also an equivalence transformation.

In particular cases of arbitrary elements the equivalence Lie group can be extended [7]. Extensions of the equivalence Lie group (5) only occur for particular choices of the function $H$. These extensions are presented in Table 1. Each column of the table lists the additional equivalence Lie group generators for the corresponding particular functions $H$.

Table 1: Extensions of the equivalence Lie group

\begin{tabular}{llcc}
\hline \multicolumn{1}{c}{$H=\alpha$} & $H=\alpha \varphi, \alpha \neq 0$ & $H=e^{\alpha \varphi}, \alpha \neq 0$ & $H=\beta \varphi^{\alpha}, \beta \neq 0$ \\
\hline \hline & $\varphi \partial_{\varphi}+\varphi_{s} \partial_{\varphi_{s}}$, & & \\
$t \partial_{\varphi}$, & $\sinh (\sqrt{\alpha} t) \partial_{\varphi}$ if $\alpha>0$, & $t \partial_{t}+s \partial_{s}$ & $t \partial_{t}+s \partial_{s}-\frac{2 \varphi}{\alpha-1} \partial_{\varphi}$ \\
$s \partial_{\varphi}+\partial_{\varphi_{s}}$, & $\cosh (\sqrt{\alpha} t) \partial_{\varphi}$ if $\alpha>0$, & $-\frac{2}{\alpha} \partial_{\varphi}-\varphi_{s} \partial_{\varphi_{s}}$, & $+\frac{1+\alpha}{1-\alpha} \varphi_{s} \partial_{\varphi_{s}}$, \\
$t^{2} \partial_{\varphi}+2 \partial_{\alpha}$ & $\sin (\sqrt{|\alpha|} t) \partial_{\varphi}$ if $\alpha<0$, & $(\alpha \varphi+1) \partial_{\varphi}-\alpha^{2} \partial_{\alpha}$ & $\beta \partial_{\beta}-\frac{\varphi}{\alpha-1} \partial_{\varphi}-\frac{\varphi_{s}}{\alpha-1} \partial_{\varphi_{s}}$ \\
& $\cos (\sqrt{|\alpha|} t) \partial_{\varphi}$ if $\alpha<0$ & $+\alpha \varphi_{s} \partial_{\varphi_{s}}$ & \\
\hline
\end{tabular}

In the group classification the following equivalence transformations are used: the transformation corresponding to the generator $X_{3}^{e}$

$$
\varphi \mapsto \varphi+\varepsilon_{1}
$$

the transformation corresponding to the generator $X_{4}^{e}$

$$
t \mapsto t e^{\varepsilon_{2}}, \quad s \mapsto s e^{\varepsilon_{2}}, \quad H \mapsto H e^{-2 \varepsilon_{2}} ;
$$

the transformation corresponding to generator $t^{2} \partial_{\varphi}+2 \partial_{\alpha}$

$$
\varphi \mapsto \varphi+\varepsilon_{3} t^{2}, \quad \alpha \mapsto \alpha+2 \varepsilon_{3} ;
$$

and the transformation corresponding to generator $s \partial_{\varphi}+\partial_{\varphi_{s}}$, which allows one to shift $\varphi_{s}$,

$$
s \mapsto s \varphi+\varepsilon_{4} s, \quad \varphi_{s} \mapsto \varphi_{s}+\varepsilon_{4} .
$$

\subsection{Admitted Lie group}

A generator admitted by equation (3) is considered in the form

$$
X=\xi^{t} \partial_{t}+\xi^{s} \partial_{s}+\eta \partial_{\varphi},
$$


where its coefficients depend on $(t, s, \varphi)$.

$$
\xi^{s}=C_{1} s+C_{2}, \quad \eta=\left(\frac{1}{2} \xi_{t}^{t}+C_{3}\right) \varphi+\tau_{1} s+\tau_{2},
$$

where the constants $C_{1}, C_{2}, C_{3}$ and the functions $\tau_{1}(t), \tau_{2}(t)$ and $\xi^{t}=\xi^{t}(t)$ satisfy the classifying equations

$$
\begin{aligned}
& \left(\left(\frac{1}{2} \xi_{t}^{t}+C_{3}-C_{1}\right) \varphi_{s}+\tau_{1}\right) G^{\prime}+2\left(\xi_{t}^{t}-C_{1}\right) G=0, \\
& \left(\left(\frac{1}{2} \xi_{t}^{t}+C_{3}\right) \varphi+\tau_{2}\right) H^{\prime}-\left(C_{3}-\frac{3}{2} \xi_{t}^{t}\right) H-\frac{1}{2} \xi_{t t t}^{t} \varphi-\tau_{2}^{\prime \prime}=0, \\
& \tau_{1} H^{\prime}-\tau_{1}^{\prime \prime}=0 .
\end{aligned}
$$

Notice that here and further on $C_{i}$ are constant.

The kernel of admitted Lie algebras is derived by splitting equations (77) with respect to $G, G^{\prime}, H$ and $H^{\prime}$. Its basis consists of the generators

$$
X_{1}=\partial_{t}, \quad X_{2}=\partial_{s}
$$

Extensions of the kernel of admitted Lie algebras occur for particular cases of the functions $G$ and $H$. Further these particular cases are derived.

Differentiating the third equation of system (7) by $\varphi$, one obtains

$$
\tau_{1} H^{\prime \prime}=0
$$

Hence, the analysis of system (7) is split into the cases: a linear function $H$, i.e. $H^{\prime \prime}=0$, and a nonlinear function $H$, i.e. $\tau_{1}=0$.

\subsection{The function $H$ is linear}

For linear function $H$, i.e.,

$$
H=\alpha \varphi+\beta,
$$

where $\alpha$ and $\beta$ are constant, one can assume that $\beta=0$. Indeed, if $\alpha=0$, then, using the equivalence transformations corresponding to the generator $t^{2} \partial_{\varphi}+2 \partial_{\beta}$, one can assume that $\beta=0$. If $\alpha \neq 0$ then one can reduce $\beta$ by the equivalence transformations corresponding to the generator $X_{3}^{e}$.

\subsubsection{Case of $H=0$}

Substituting $H=0$ in (7), one obtains

$$
\tau_{1}^{\prime \prime}=0, \quad \tau_{2}^{\prime \prime}=0, \quad \xi_{t t t}^{t}=0
$$

It follows that

$$
\xi^{t}=K_{1} t^{2}+K_{2} t+K_{3}, \quad \tau_{1}=A_{1} t+A_{2}, \quad \tau_{2}=B_{1} t+B_{2},
$$

where $A_{i}, B_{i}, K_{i}$ are constant. Substituting the latter relations into (77) and splitting by $t$, one derives that

$$
\begin{gathered}
\left(K_{1} \varphi_{s}+A_{1}\right) G^{\prime}+4 K_{1} G=0 \\
\left(\left(\frac{1}{2} K_{2}+C_{3}-C_{1}\right) \varphi_{s}+A_{2}\right) G^{\prime}+2\left(K_{2}-C_{1}\right) G=0 .
\end{gathered}
$$


For an arbitrary function $G$ one obtains that

$$
K_{1}=0, \quad A_{1}=0, \quad A_{2}=0, \quad K_{2}=C_{1}=2 C_{3},
$$

and the extension of the kernel (8) is defined by the generators

$$
\partial_{\varphi}, \quad t \partial_{\varphi}, \quad t \partial_{t}+s \partial_{s}+\varphi \partial_{\varphi}
$$

From system (9) one can conclude that there exist constants $a, b$ and $c$ such that

$$
\left(a \varphi_{s}+b\right) G^{\prime}+c G=0
$$

By virtue of the equivalence transformations, for finding extensions of (10) one needs to study two representations of the functions $G$.

The first form of $G$ is

$$
G=-e^{\mu \varphi_{s}},
$$

where $\mu \neq 0$ is constant. Substituting $G$ into (9), one obtains that

$$
K_{1}=0, \quad A_{1}=0, \quad K 2=2\left(C_{1}-C_{3}\right), \quad 2\left(C_{1}-2 C_{3}\right)+\mu A_{2}=0 .
$$

The extension of (10) is defined by the generator

$$
t \partial_{t}-\frac{2 s}{\mu} \partial_{\varphi}
$$

The second form of the function $G$ is defined by the generators

$$
G=-\varphi_{s}^{\lambda}
$$

where $\lambda$ is constant such that $\lambda(1-\lambda) \neq 0$. Substituting $G$ into (9), one obtains that

$$
A_{1}=0, \quad A_{2}=0, \quad K_{1}(\lambda+4)=0, \quad(\lambda+4) K_{2}-2 C_{1}(\lambda+2)+2 \lambda C_{3}=0 .
$$

If $\lambda=-4$, then $C_{1}=2 C_{3}$, and the extension of (10) is

$$
2 t \partial_{t}+\varphi \partial_{\varphi} \quad t^{2} \partial_{t}+t \varphi \partial_{\varphi}
$$

If $\lambda=-2$, then $K_{1}=0$ and $K_{2}=2 C_{3}$. The extension of (8) is

$$
s \partial_{s}, \quad t \partial_{t}+\varphi \partial_{\varphi}
$$

If $(\lambda+4)(\lambda+2) \neq 0$, then

$$
K_{1}=0, \quad K_{2}=\frac{2 C_{1}(\lambda+2)-2 \lambda C_{3}}{\lambda+4},
$$

and the extension of (10) is

$$
t \partial_{t}-\frac{2}{\lambda} \varphi \partial_{\varphi}
$$




\subsubsection{Case of $H=\alpha \varphi, \alpha \neq 0$}

It follows from system (7) that

$$
\begin{aligned}
& \left(\left(\frac{1}{2} \xi_{t}^{t}+C_{3}-C_{1}\right) \varphi_{s}+\tau_{1}\right) G^{\prime}+2\left(\xi_{t}^{t}-C_{1}\right) G=0, \\
& \xi_{t t t}^{t}=4 \alpha \xi_{t}^{t}, \quad \tau_{1}^{\prime \prime}=\alpha \tau_{1} \quad \tau_{2}^{\prime \prime}=\alpha \tau_{2} .
\end{aligned}
$$

If $G$ is an arbitrary function, then

$$
\xi_{t}^{t}=C_{1}=C_{3}=0, \quad \tau_{1}=0,
$$

The extension of (8) is defined by the generators

$$
\begin{array}{lll}
\sin \sqrt{|\alpha|} t \partial_{\varphi}, & \cos \sqrt{|\alpha|} t \partial_{\varphi}, & \alpha<0, \\
\cosh \sqrt{\alpha} t \partial_{\varphi}, & \sinh \sqrt{\alpha} t \partial_{\varphi}, & \alpha>0 .
\end{array}
$$

As in the previous case, one has to study two forms of the function $G$. The first form is

$$
G=-e^{\mu \varphi_{s}},
$$

where $\mu \neq 0$ is constant. Substituting $G$ into (7), one gets

$$
C_{1}=C_{3}=0, \quad \xi_{t}^{t}=0, \quad \tau_{1}=0, \quad \tau_{2}^{\prime \prime}-\alpha \tau_{2}=0 .
$$

In this case there is no an extension of (13).

Another form of $G$ is

$$
G=-\left(\varphi_{s}+c\right)^{\lambda},
$$

where $\lambda$ and $c$ are constant, such that $\lambda(1-\lambda) \neq 0$. Substituting $G$ into the first equation of (7), one obtains

$$
(\lambda+4) \xi_{t}^{t}-2(\lambda+2) C_{1}+2 \lambda C_{3}=0, \quad \tau_{1}=\frac{2 c\left(\xi_{t}^{t}-C_{1}\right)}{\lambda}, \quad c\left(3 \xi_{t}^{t}+C_{1}\right)=0 .
$$

If $c \neq 0$ then

$$
\xi_{t}^{t}=0, \quad \tau_{1}=0, \quad C_{1}=0, \quad C_{3}=0,
$$

and there is no an extension of (13) in this case.

If $c=0$, then $\tau_{1}=0$. Integrating the last three equations of system (12), one obtains

$$
\begin{aligned}
& \xi^{t}=A_{3}+ \begin{cases}A_{1} \sin 2 \sqrt{|\alpha|} t+A_{2} \cos 2 \sqrt{|\alpha|} t, & \alpha<0, \\
A_{1} \sinh 2 \sqrt{\alpha} t+A_{2} \cosh 2 \sqrt{\alpha} t, & \alpha>0 ;\end{cases} \\
& \tau_{2}= \begin{cases}B_{1} \sin \sqrt{|\alpha|} t+B_{2} \cos \sqrt{|\alpha|} t, & \alpha<0, \\
B_{1} \sinh \sqrt{\alpha} t+B_{2} \cosh \sqrt{\alpha} t, & \alpha>0,\end{cases}
\end{aligned}
$$

where $A_{i}, B_{i}$ are constant. Substituting (14) into the first equation of system (12), one derives that

$$
B_{1}=0, \quad B_{2}=0, \quad \sqrt{|\alpha|}(\lambda+4) A_{1}+C_{1}(2+\lambda)-\lambda C_{3}=0,
$$


and

$$
\begin{gathered}
(\lambda+4) \sqrt{|\alpha|}\left(A_{2} \sin 2 \sqrt{|\alpha|} t-2 A_{1} \cos ^{2} \sqrt{|\alpha|} t\right)=0, \quad \alpha<0 ; \\
(\lambda+4) \sqrt{\alpha}\left(A_{2} \sinh 2 \sqrt{\alpha} t-2 A_{1} \cosh ^{2} \sqrt{\alpha} t\right)=0, \quad \alpha>0 .
\end{gathered}
$$

Then, for any $\lambda \neq 0$ the following extension of (13) occurs

$$
s \partial_{s}+\frac{2+\lambda}{\lambda} \varphi \partial_{\varphi} .
$$

If $\lambda=-4$, then there are additional to (13) generators

$$
\begin{gathered}
\sin 2 \sqrt{|\alpha|} t \partial_{t}+\sqrt{|\alpha|} \varphi \cos 2 \sqrt{|\alpha|} t \partial_{\varphi}, \quad \alpha<0 \\
\sinh 2 \sqrt{\alpha} t \partial_{t}+\sqrt{\alpha} \varphi \cosh 2 \sqrt{\alpha} t \partial_{\varphi}, \quad \alpha>0
\end{gathered}
$$

and

$$
\begin{gathered}
\cos 2 \sqrt{|\alpha|} t \partial_{t}-\sqrt{|\alpha|} \varphi \sin 2 \sqrt{|\alpha|} t \partial_{\varphi}, \quad \alpha<0 \\
\cosh 2 \sqrt{\alpha} t \partial_{t}+\sqrt{\alpha} \varphi \sinh 2 \sqrt{\alpha} t \partial_{\varphi}, \quad \alpha>0 .
\end{gathered}
$$

\subsection{The function $H$ is nonlinear}

As mentioned for the nonlinear function $H$ one has that $\tau_{1}=0$. System (7) becomes

$$
\begin{aligned}
& \left(\frac{1}{2} \xi_{t}^{t}+C_{3}-C_{1}\right) \varphi_{s} \frac{G^{\prime}}{G}+2\left(\xi_{t}^{t}-C_{1}\right)=0, \\
& \left(\left(\frac{1}{2} \xi_{t}^{t}+C_{3}\right) \varphi+\tau_{2}\right) H^{\prime}-\left(C_{3}-\frac{3}{2} \xi_{t}^{t}\right) H-\frac{1}{2} \xi_{t t t}^{t} \varphi-\tau_{2}^{\prime \prime}=0 .
\end{aligned}
$$

Recall that

$$
\xi^{t}=\xi^{t}(t), \quad \xi^{s}=C_{1} s+C_{2}, \quad \eta=\left(\frac{1}{2} \xi_{t}^{t}+C_{3}\right) \varphi+\tau_{2}(t) .
$$

Differentiating the first equation of system (15) with respect to $\varphi_{s}$, one obtains

$$
\left(\frac{1}{2} \xi_{t}^{t}+C_{3}-C_{1}\right)\left(\varphi_{s} \frac{G^{\prime}}{G}\right)^{\prime}=0
$$

Case of $\left(\varphi_{s} \frac{G^{\prime}}{G}\right)^{\prime} \neq 0$. The latter equation and the first equation of (15) gives that

$$
\xi_{t}^{t}=C_{1}, \quad C_{3}=C_{1} / 2 .
$$

The second equation of (15) becomes

$$
\left(C_{1} \varphi+\tau_{2}\right) H^{\prime}+C_{1} H-\tau_{2}^{\prime \prime}=0 .
$$

Differentiating the latter equation by $\varphi$ and then by $t$, one gets that $\tau_{2}^{\prime} H^{\prime \prime}=0$, which gives that $\tau_{2}$ is constant, and

$$
\left(C_{1} \varphi+\tau_{2}\right) H^{\prime}+C_{1} H=0,
$$

Notice, that, if $C_{1}=0$, then for the nonlinear $H$, one has that $\tau_{2}=0$, which does not lead to an extension of the kernel (8). Hence, one needs to consider the case $C_{1} \neq 0$. By virtue of the equivalence transformations one can assume that the function $H$ has the form

$$
H=\beta \varphi^{-1},
$$

where $\beta \neq 0$ is constant. Substituting the latter relation into (16), one derives that $\tau_{2}=0$, and $G$ is an arbitrary nonlinear function. Thus, for $\left(\varphi_{s} \frac{G^{\prime}}{G}\right)^{\prime} \neq 0$ the extension of (18) is given by the generator

$$
t \partial_{t}+s \partial_{s}+\varphi \partial_{\varphi}
$$


Case of $\left(\varphi_{s} \frac{G^{\prime}}{G}\right)^{\prime}=0$. Here

$$
G=-\left(\varphi_{s}+c\right)^{\lambda}
$$

where $c$ and $\lambda \neq 0$ are constant. By virtue of the nonlinearity of $G$, one has that $(\lambda-1) \lambda \neq 0$.

Substituting the latter relation into the first equation of system (15), one derives the following system of equations

$$
\begin{gathered}
c\left(\xi_{t}^{t}-C_{1}\right)=0, \\
(\lambda+4) \xi_{t}^{t}-2 C_{1}(\lambda+2)+2 \lambda C_{3}=0 .
\end{gathered}
$$

If $c \neq 0$, then $\xi_{t}^{t}=C_{1}, C_{3}=C_{1} / 2$, and the equation for $H$ becomes (16). This case was studied above.

Hence, one needs to consider $c=0$, i.e. the function $G=-\varphi^{\lambda}$.

Differentiating the second equation of system (15) twice by $\varphi$, and then by $t$, one gets

$$
5 \xi_{t t}^{t} H^{\prime \prime}+\left(\xi_{t t}^{t} \varphi+2 \tau_{2}^{\prime}\right) H^{\prime \prime \prime}=0
$$

First we analyze the case $H^{\prime \prime \prime} \neq 0$. Differentiating (18) by $\varphi$, one obtains

$$
\xi_{t t}^{t}\left(5\left(\frac{H^{\prime \prime}}{H^{\prime \prime \prime}}\right)^{\prime}+1\right)=0 .
$$

Thus, one ends up with the following cases.

Case $\left(\frac{H^{\prime \prime}}{H^{\prime \prime \prime}}\right)^{\prime}=-1 / 5$. Integrating one finds that

$$
H=\alpha(\varphi+\delta)^{-3}+\beta \varphi+\varepsilon
$$

where $\alpha \neq 0, \beta$ and $\varepsilon, \delta$ are constant. By virtue of the equivalence transformation corresponding to the generator $X_{3}^{e}$ one can assume $\delta=0$.

Substituting $H$ into system (15), one gets

$$
\begin{gathered}
(\lambda+4) \xi_{t t}^{t}+2\left(C_{3}-C_{1}\right) \lambda-4 C_{1}=0, \\
\left(\xi_{t t t}^{t}-4 \beta \xi_{t}^{t}\right) \varphi+2 \alpha\left(4 C_{3} \varphi^{-3}+3 \tau_{2} \varphi^{-4}\right)+\varepsilon\left(2 C_{3}-3 \xi_{t}^{t}\right)+2\left(\tau_{2}^{\prime \prime}-\beta \tau_{2}\right)=0 .
\end{gathered}
$$

Splitting the latter equation with respect to $\varphi$, system of equations (17), (20) reduces to the equations

$$
\begin{gathered}
C_{3}=0, \quad \tau_{2}=0, \\
(\lambda+4) \xi_{t}^{t}-2(\lambda+2) C_{1}=0, \\
\xi_{t t t}^{t}-4 \beta \xi_{t}^{t}=0, \quad \varepsilon \xi_{t}^{t}=0 .
\end{gathered}
$$

Notice that if $\varepsilon \neq 0$, then extension of (8) only occurs for $\lambda=-2$, and it is defined by the generator $s \partial_{s}$. Hence, it is assumed that $\varepsilon=0$.

(i) If $\lambda=-4$, then $C_{1}=0$, and system (21) gives that $\xi^{t}$ must satisfy the following equation

$$
\xi_{t t t}^{t}-4 \beta \xi_{t}^{t}=0
$$


If $\beta=0$, then $\xi_{t t t}^{t}=0$ and one derives that the extension of (8) is

$$
\partial_{\varphi}, \quad t \partial_{\varphi}, \quad 2 t \partial_{t}+\varphi \partial_{\varphi} \quad t^{2} \partial_{t}+2 t \varphi \partial_{\varphi}
$$

If $\beta \neq 0$, then the extensions of (8) are

$$
\begin{gathered}
\sin 2 \sqrt{|\beta|} t \partial_{t}+\sqrt{|\beta|} \varphi \cos 2 \sqrt{|\beta|} t \partial_{\varphi}, \beta<0, \\
\sinh 2 \sqrt{\beta} t \partial_{t}+\sqrt{\beta} \varphi \cosh 2 \sqrt{\beta} t \partial_{\varphi}, \beta>0,
\end{gathered}
$$

and

$$
\begin{gathered}
\cos 2 \sqrt{|\beta|} t \partial_{t}-\sqrt{|\beta|} \varphi \sin 2 \sqrt{|\beta|} t \partial_{\varphi}, \beta<0, \\
\cosh 2 \sqrt{\beta} t \partial_{t}+\sqrt{\beta} \varphi \sinh 2 \sqrt{\beta} t \partial_{\varphi}, \beta>0 .
\end{gathered}
$$

Notice, that for $H=\alpha \varphi^{-3}+\beta \varphi$ and $G=-\varphi^{-4}$ the extensions coincide with the case where $\alpha=0$.

(ii) If $\lambda=-2$, then the extension of (8) is defined by the generator $s \partial_{s}$.

(iii) If $(\lambda+2)(\lambda+4) \neq 0$, then, form (21), one gets

$$
\xi_{t}^{t}=2 C_{1} \frac{\lambda+2}{\lambda+4}, \quad \beta C_{1}(\lambda+2)=0 .
$$

The extension of (8) only occurs for $\beta=0$ :

$$
2 t \partial_{t}+\frac{\lambda+4}{\lambda+2} s \partial_{s}+\varphi \partial_{\varphi}
$$

Case of $\left(\frac{H^{\prime \prime}}{H^{\prime \prime \prime}}\right)^{\prime} \neq-1 / 5 . \quad$ Recall that in this case $\xi_{t t}^{t}=0$, i.e.,

$$
\xi^{t}=A_{1} t+A_{2}
$$

where $A_{i}$ are constant. System (15) becomes

$$
\begin{gathered}
(\lambda+4) A_{1}-2(\lambda+2) C_{1}+\lambda C_{3}=0, \\
\left(\left(A_{1}+2 C_{3}\right) \varphi+2 \tau_{2}\right) H^{\prime}+\left(3 A_{1}-2 C_{3}\right) H-2 \tau_{2}^{\prime \prime}=0 .
\end{gathered}
$$

Differentiating the second equation of (22) with respect to $\varphi$ and $t$, one obtains that $\tau_{2}$ is constant, and analysis of the second equation of (22) leads to the study of two types of the function: $H=e^{\alpha \varphi}$, where $\alpha \neq 0$, and $H=\beta \varphi^{\alpha}$, where $\alpha(1-\alpha) \neq 0$.

(i) If $H=e^{\alpha \varphi}$, then system (22) reduces to the equations

$$
(\lambda+2) C_{1}+4 C_{3}=0, \quad A_{1}=-2 C_{3}, \quad \alpha \tau_{2}=4 C_{3} .
$$

If $\lambda \neq-2$, then $\tau_{2}=4 C_{3} / \alpha$ and $C_{3}=-C_{1}(\lambda+2) / 4$. Hence, the extension of (8) is

$$
t \partial_{t}+\frac{2}{2+\lambda} s \partial_{s}-\frac{2}{\alpha} \partial_{\varphi} .
$$

If $\lambda=-2$, then $C_{3}=0$ and $\tau_{2}=0$, and the only extension of the kernel (8) is given by the generator $s \partial_{s}$. 
(ii) In case of $H=\beta \varphi^{\alpha}$, one notices that from the condition $\left(\frac{H^{\prime \prime}}{H^{\prime \prime \prime}}\right)^{\prime} \neq-1 / 5$ implies that $\alpha \neq-3$.

The second equation of system (22) leads to the equations

$$
\tau_{2}=0, \quad A_{1}=2 \frac{1-\alpha}{3+\alpha} C_{3}
$$

If $\lambda \neq-2$, then

$$
C_{1}=\frac{4 C_{3}(\lambda-\alpha+1)}{(\lambda+2)(\alpha+3)},
$$

and the extension of the kernel (8) is defined by the generator

$$
t \partial_{t}+\frac{2(\alpha-\lambda-1)}{(2+\lambda)(\alpha-1)} s \partial_{s}+\frac{2 \varphi}{1-\alpha} \partial_{\varphi} .
$$

If $\lambda=-2$, then the first equation of system (22) reduces to

$$
C_{3}(\alpha+1)=0
$$

If $\alpha \neq-1$, then $C_{3}=0$, and the extension of the kernel (8) is defined by the generator $s \partial_{s}$. If $\alpha=-1$, then the extension of (요 is

$$
s \partial_{s}, \quad t \partial_{t}+\varphi \partial_{\varphi}
$$

Case of $H^{\prime \prime \prime}=0$. In this case it follows that

$$
H=\alpha \varphi^{2}+\beta \varphi+\delta
$$

where $\alpha \neq 0, \beta, \delta$ are constant, and the constant $\delta$ can be vanished by equivalence transformations.

Splitting the second equation of (15), and using the second equation of (17), one obtains

$$
\xi_{t}^{t}=2 \frac{\lambda+2}{1-\lambda} C_{1}, \quad C_{3}=-5 \frac{\lambda+2}{1-\lambda} C_{1}, \quad \tau_{2}=0 .
$$

In this case the extension of the kernel (8) is defined by the generator

$$
(\lambda+2)\left(t \partial_{t}-2 \varphi \partial_{\varphi}\right)+2(1-\lambda) s \partial_{s}
$$

Notice that this is just a particular case of (23).

Results of the presented above classifications are given in Table 2 and Table 3 , Table 2 contains extensions of the kernel of admitted Lie algebras, where one of the functions, either $G$ or $H$, is arbitrary. The columns of the table correspond to different functions $H$, and the rows correspond to different functions $G$. On the intersection of the columns and the rows the extensions of (8) are presented. Generators provided in Table 3 extend the contents of Table 2, Constraints on the values of constants are included in the table.

Remark. A polytropic gas corresponds to the function $G=-\varphi_{s}^{\lambda}[\underline{5]}$, where $\lambda$ and the polytropic exponent $\gamma$ are related by the formula $\lambda=-\gamma-1$. In particular, for the hyperbolic shallow water equations $\lambda=-3[6]$. 
Table 2: Extensions of [8) (arbitrary $G$ or $H$ ).

\begin{tabular}{c|clcl}
\hline & $H=H(\varphi)$ & $H=0$ & $H=\alpha \varphi, \alpha \neq 0$ & $H=\beta \varphi^{-1}, \beta \neq 0$ \\
\hline \hline \multirow{2}{*}{$G=G\left(\varphi_{s}\right)$} & $X_{3}=\partial_{\varphi}$, & $X_{3}= \begin{cases}\sin \sqrt{|\alpha|} t \partial_{\varphi}, & \alpha<0, \\
\sinh \sqrt{\alpha} t \partial_{\varphi}, & \alpha>0,\end{cases}$ \\
& $X_{2}=\partial_{s}$ & $X_{4}=t \partial_{\varphi}$, & $X_{5}=t \partial_{t}+s \partial_{s}+\varphi \partial_{\varphi}$ & $X_{4}+s \partial_{s}+\varphi \partial_{\varphi}$. \\
$\cos \sqrt{|\alpha|} t \partial_{\varphi}$, & $\alpha>0$, \\
$\cosh \sqrt{\alpha} t \partial_{\varphi}$, & $\alpha>0$.
\end{tabular}

Table 3: Extensions of (8).

\begin{tabular}{|c|c|c|c|c|}
\hline & $H=0$ & $\begin{array}{c}H=\beta \varphi^{-3}+\alpha \varphi \\
\alpha \neq 0\end{array}$ & $\begin{array}{c}H=e^{\alpha \varphi} \\
\alpha \neq 0\end{array}$ & $\begin{array}{c}H=\beta \varphi^{\alpha} \\
\alpha \neq 0,1, \beta \neq 0\end{array}$ \\
\hline $\begin{array}{l}G=-e^{\mu \varphi_{s}}, \\
\mu \neq 0\end{array}$ & $X_{6}=t \partial_{t}-\frac{2}{\mu} s \partial_{\varphi}$ & - & - & - \\
\hline $\begin{array}{c}G=-\varphi_{s}^{\lambda} \\
\lambda \neq 0,1\end{array}$ & $\begin{array}{l}X_{6}=t \partial_{t}-\frac{2}{\lambda} \varphi \partial_{\varphi} \\
\text { if } \lambda=-4: \\
X_{7}=t^{2} \partial_{t}+t \varphi \partial_{\varphi}\end{array}$ & $\begin{array}{l}\text { if } \beta=0 \\
X_{5}=s \partial_{s}+\frac{1}{\lambda}(2+\lambda) \varphi \partial_{\varphi} \\
\text { if } \lambda=-4: \\
X_{6}=\left\{\begin{array}{l}\sin 2 \sqrt{|\alpha|} t \partial_{t}+\sqrt{|\alpha|} \varphi \cos 2 \sqrt{|\alpha|} t \partial_{\varphi}, \alpha<0 \\
\sinh 2 \sqrt{\alpha} t \partial_{t}+\sqrt{\alpha} \varphi \cosh 2 \sqrt{\alpha} t \partial_{\varphi}, \alpha>0\end{array}\right. \\
X_{7}=\left\{\begin{array}{l}\cos 2 \sqrt{|\alpha|} t \partial_{t}-\sqrt{|\alpha|} \varphi \sin 2 \sqrt{|\alpha|} t \partial_{\varphi}, \alpha<0 \\
\cosh 2 \sqrt{\alpha} t \partial_{t}+\sqrt{\alpha} \varphi \sinh 2 \sqrt{\alpha} t \partial_{\varphi}, \alpha>0\end{array}\right.\end{array}$ & $\begin{array}{l}\text { if } \lambda \neq-2: \\
\begin{aligned} X_{3} & =t \partial_{t} \\
+ & \frac{2}{2+\lambda} s \partial_{s} \\
& -\frac{2}{\alpha} \partial_{\varphi}\end{aligned}\end{array}$ & $\begin{array}{l}\text { if } \lambda \neq-2: \\
\begin{array}{r}X_{3}=t \partial t \\
\quad+\frac{2(\alpha-\lambda-1)}{(2+\lambda)(\alpha-1)} s \partial_{s} \\
\quad+\frac{2 \varphi}{1-\alpha} \partial_{\varphi} .\end{array} \\
\text { if } \lambda=-4, \alpha=-3 \\
X_{4}=t^{2} \partial_{t}+t \varphi \partial_{\varphi}\end{array}$ \\
\hline
\end{tabular}

\section{Conservation laws}

Noether's theorem allows one to find conservation laws for the obtained equations using the Lagrangian (2) and the found admitted symmetries [4]. The theorem states that if a Lagrangian $\mathcal{L}$ is invariant of the action of a symmetry $X$, i.e.,

$$
X \mathcal{L}+\mathcal{L}\left(D_{t} \xi^{t}+D_{s} \xi^{s}\right)=D_{t}\left(V^{t}\right)+D_{s}\left(V^{s}\right)
$$

then the Euler-Lagrange equation (3) possesses the following conservation law

$$
D_{t}\left(T^{t}\right)+D_{s}\left(T^{s}\right)=D_{t}\left(\xi^{t} \mathcal{L}+\zeta \frac{\partial \mathcal{L}}{\partial \varphi_{t}}-V^{t}\right)+D_{s}\left(\xi^{s} \mathcal{L}+\zeta \frac{\partial \mathcal{L}}{\partial \varphi_{s}}-V^{s}\right)=0
$$

where $\zeta=\eta-\xi^{t} \varphi_{t}-\xi^{s} \varphi_{s}$.

If the vector $\left(V^{t}, V^{s}\right)$ is not a zero vector, the conservation law is also called a divergent one.

Conservation laws in Lagrangian coordinates and their representations in Eulerian coordinates are listed in Table 4 and Table 5 respectively. Note also that there are conservation laws in Lagrange coordinates which have no representations in Eulerian coordinates.

\section{Conclusion}

Comprehensive analysis of equation (3) is given in the present paper. Equation (3) describes a motion of continuum (1) in Lagrangian coordinates. It contains two arbitrary functions $G\left(\varphi_{s}\right)$ and $H(\varphi)$. Particular 
choices of these functions correspond to different models studied in continuum mechanics, such as, isentropic flows of an ideal gas [5], different types of hyperbolic shallow water equations [9, 17]. One of the objectives of the present paper is to derive conservation laws of a continuum defined by equation (3).

As equation (3) is an Euler-Lagrange equation, for constructing conservation laws we used Noether's theorem. To apply Noether's theorem, first complete group analysis of equation (3) is performed. Results of the group classification are given in Table 2 and Table 3. The group classification separates out the nonlinear function $G$ into the following forms: either the function $G$ is arbitrary or it has the exponential form $-e^{\mu \varphi_{s}}$ or the polynomial form $-\varphi_{s}^{\lambda}$, where $\mu$ and $\lambda$ are constant. All extensions of the kernels of admitted Lie algebras are found. These extensions occur for a particular cases of the function $H$.

Second using Noether's theorem, the conservation laws of equation (4) were obtained in Lagrangian. Their representations in Eulerian coordinates were also presented. It should be noted that some of the conservation laws have no their counterpart in Eulerian coordinates. All found conservation laws are listed in Table 4 and Table 5 .

\section{Acknowledgements}

The research was supported by Russian Science Foundation Grant No 18-11-00238 'Hydrodynamics-type equations: symmetries, conservation laws, invariant difference schemes'. E.I.K. also acknowledges Suranaree University of Technology for Full-time Master Researcher Fellowship (15/2561). The authors thank V.A.Dorodnitsyn and E.Schulz for valuable discussions.

\section{References}

[1] L. I. Sedov. Continuum mechanics, v. 1, 5-th ed. Nauka, Moscow, 1994. in Russian.

[2] S. L. Gavrilyuk and S. M. Shugrin. Media with equations of state that depend on derivatives. Journal of Applied Mechanics and Technical Physics, 37(2):177-189, 1996.

[3] S. L. Gavrilyuk and V. M. Teshukov. Generalized vorticity for bubbly liquid and dispersive shallow water equations. Continuum Mech. Thermodyn., 13:365-382, 2001.

[4] N. H. Ibragimov. Transformation Groups Applied to Mathematical Physics. Nauka, Moscow, 1983. English translation, Reidel, D., Ed., Dordrecht, 1985.

[5] G. G. Chernyi. Gas dynamics. Nauka, Moscow, 1988. in Russian.

[6] P. Siriwat, C. Kaewmanee, and S. V. Meleshko. Symmetries of the hyperbolic shallow water equations and the Green-Naghdi model in Lagrangian coordinates. International Journal of Non-Linear Mechanics, 86:185-195, 2016.

[7] L. V. Ovsiannikov. Group analysis of differential equations. Nauka, Moscow, 1978. English translation, Ames, W.F., Ed., published by Academic Press, New York, 1982.

[8] Yu. A. Chirkunov, S. Yu. Dobrokhotov, S. B. Medvedev, and D. S. Minenkov. Exact solutions of one-dimensional nonlinear shallow water equations over even and slopic bottoms. Theoretical and Mathematical Physics, 178(3):278-298, 2014.

[9] S. Szatmari and A. Bihlo. Symmetry analysis of a system of modified shallow-water equations. Communications in Nonlinear Science and Numerical Simulation, 19:530-537, 2014.

[10] A. V. Aksenov and K. P. Druzhkov. Conservation laws, symmetries and exact solutions of the shallow water system of equations above not straight bottom. Vestnik natsional'nogo issledovatel'skogo yadernogo universiteta "MIFI", 5(1):38-46, 2016. in Russian.

[11] N. H. Ibragimov, editor. CRC Handbook of Lie Group Analysis of Differential Equations, volume 1. CRC Press, Boca Raton, 1994 . 
[12] V. A. Vinokurov and I. G. Nurgalieva. Investigation of nonlinear equation of adiabatic motion of an ideal gas. In V. N. Vragov, editor, Nonclassical Equations of Mathematical Physics, page 53. Novosibirsk, 1985.

[13] V. K. Andreev, O.V. Kaptsov, V.V. Pukhnachov, and A. A. Rodionov. Applications of Group-Theoretic Methods in Hydrodynamics. Kluwer, Dordrecht, 1998.

[14] I. S. Akhatov, R. K. Gazizov, and N. H. Ibragimov. Nonlocal symmetries. Heuristic approach. J. Math. Sci., 55(1):1401-1450, 1991. Journal of Soviet Mathematics (in Russian).

[15] G. M. Webb and G. P. Zank. Scaling symmetries, conservation laws and action principles in one-dimensional gas dynamics. Journal of Physics A: Mathematical and Theoretical, 42, 2009. Paper 475205.

[16] A. Sjöberg and F. M. Mahomed. Non-local symmetries and conservation laws for one-dimensional gas dynamics equations. Applied Mathematics and Computation, 150:379397, 2004.

[17] G. B. Whitham. Linear and Nonlinear Waves. Wiley, New York, 1974. 
Table 4: Conservation laws in Lagrangian coordinates

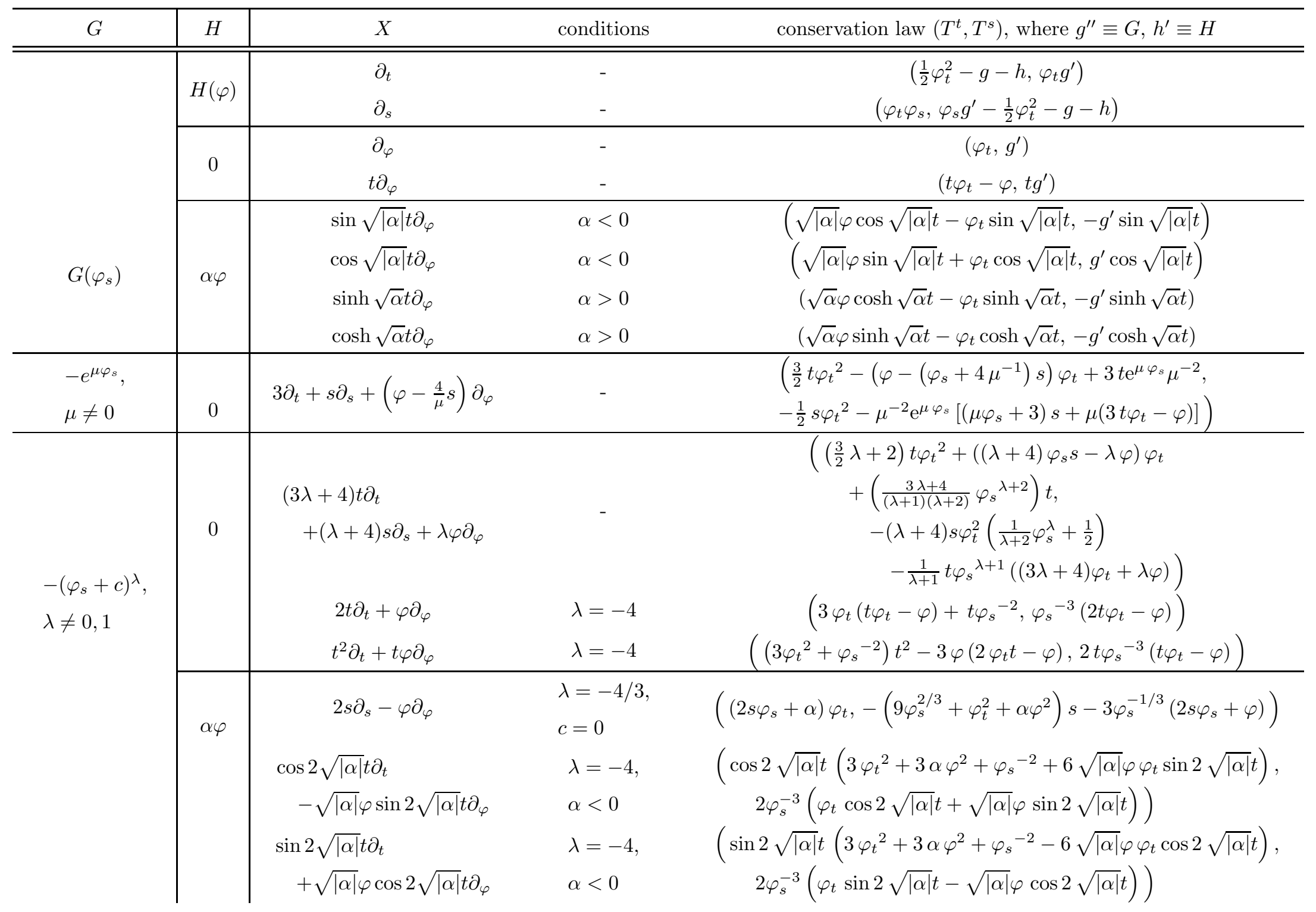


Table 4 - Conservation laws in Lagrangian coordinates (continued)

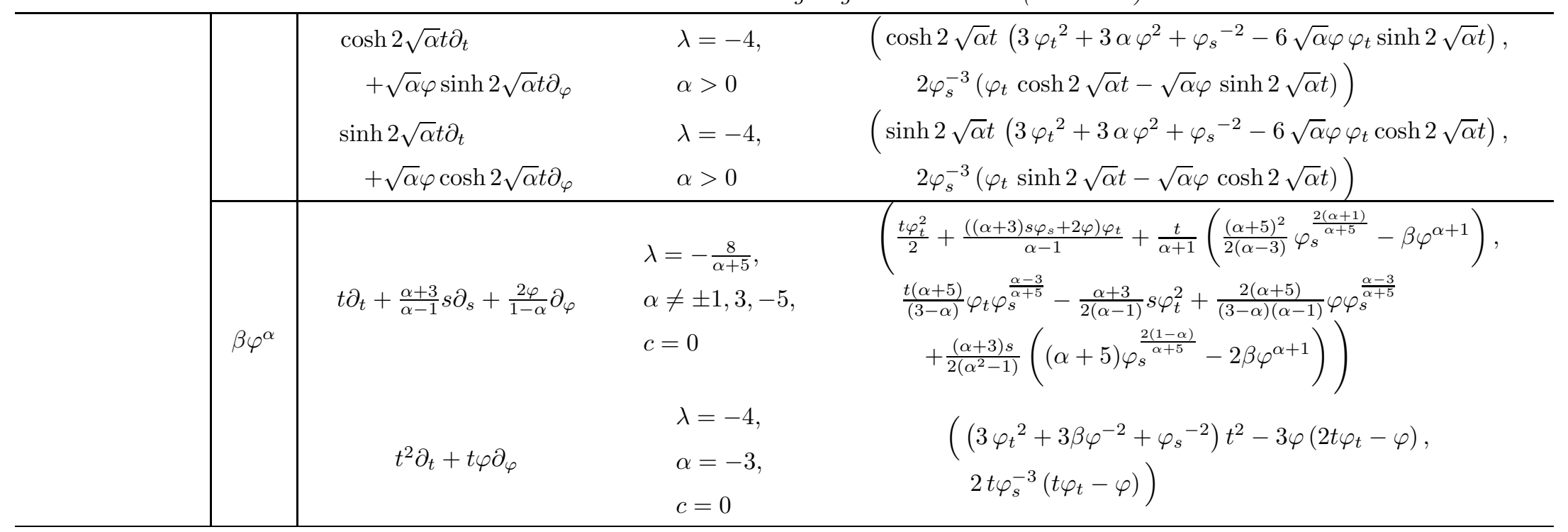

Table 5: Conservation laws in Eulerian coordinates

\begin{tabular}{|c|c|c|c|c|}
\hline$G$ & $H$ & $X$ & conditions & conservation law $\left(T^{t}, T^{x}\right)$, where $g^{\prime \prime} \equiv G, h^{\prime} \equiv H$ \\
\hline \multirow{3}{*}{$G\left(\frac{1}{\rho}\right)$} & $H(x)$ & $\begin{array}{l}\partial_{t} \\
\partial_{s}\end{array}$ & - & $\begin{array}{c}\left(\rho\left(u^{2}-2 g-2 h\right), 2 u\left(g^{\prime}+\left(\frac{1}{2} u^{2}-g-h\right) \rho\right)\right) \\
\left(u, \frac{1}{2} u^{2}-g-h+\rho^{-1} g^{\prime}\right)\end{array}$ \\
\hline & 0 & $\begin{array}{l}\partial_{\varphi} \\
t \partial_{\varphi}\end{array}$ & - & $\begin{array}{c}\left(\rho u, \rho u^{2}+g^{\prime}\right) \\
\left(\rho(x-t u), u \rho(x-t u)-t g^{\prime}\right)\end{array}$ \\
\hline & $\alpha \varphi$ & $\begin{array}{l}\sin \sqrt{|\alpha|} t \partial_{\varphi} \\
\cos \sqrt{|\alpha|} t \partial_{\varphi} \\
\sinh \sqrt{\alpha} t \partial_{\varphi}\end{array}$ & $\begin{array}{l}\alpha<0 \\
\alpha<0 \\
\alpha>0\end{array}$ & $\begin{array}{l}(\rho(\sqrt{|\alpha|} x \cos \sqrt{|\alpha|} t-u \sin \sqrt{|\alpha|} t), \\
\left.\quad x \rho u \sqrt{|\alpha|} \cos \sqrt{|\alpha|} t-\left(u^{2} \rho+g^{\prime}\right) \sin \sqrt{|\alpha|} t\right) \\
(\rho(\sqrt{|\alpha|} x \sin \sqrt{|\alpha|} t+u \cos \sqrt{|\alpha|} t), \\
\left.\quad x \rho u \sqrt{|\alpha|} \sin \sqrt{|\alpha|} t+\left(u^{2} \rho+g^{\prime}\right) \cos \sqrt{|\alpha|} t\right) \\
(\rho(\sqrt{\alpha} x \cosh \sqrt{\alpha} t-u \sinh \sqrt{\alpha} t), \\
\left.\quad x \rho u \sqrt{\alpha} \cosh \sqrt{\alpha} t-\left(u^{2} \rho+g^{\prime}\right) \sinh \sqrt{\alpha} t\right)\end{array}$ \\
\hline
\end{tabular}


Table 5 - Conservation laws in Eulerian coordinates (continued)

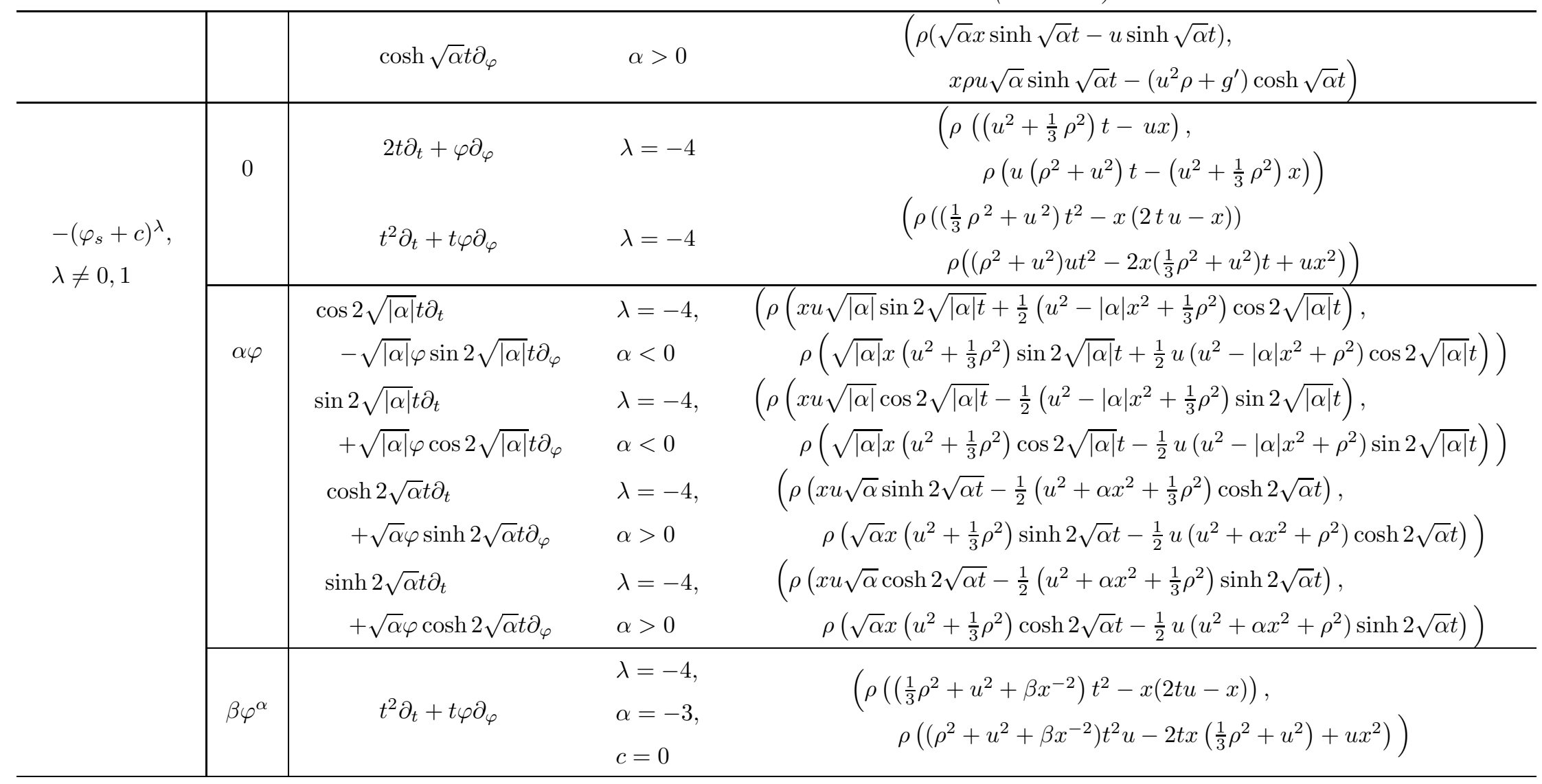

